\title{
Telomerase Reverse Transcriptase Mediated Immortalization of Human Bone Marrow Stromal Cells
}

\author{
Yong Teng ${ }^{1 *}$, YunYu Hu${ }^{2}$, Xu Sheng $\mathrm{Li}^{1}$, Zhen Wang ${ }^{2}$ and Rui Wang ${ }^{1}$ \\ ${ }^{I}$ Orthopedic Center; Orthopedic Center of Urumqi General Hospital of PLA; Lanzhou Military Region; Xinjiang \\ Uygur Autonomous Region - People's Republic of China. ${ }^{2}$ Orthopedic Institute of PLA; Xijing Hospital; The Fourth \\ Military Medical University; Xi'an - People's Republic of China
}

\begin{abstract}
Primary human bone marrow stromal cells (hMSCs) were transfected with human telomerase reverse transcriptase (hTERT) gene with lipofection method. The hTERT transfected hMSCs of passage 100 underwent chondrogenesis induction with dexamethasone, transforming the growth factor $\beta$ and vitamin $C$, osteogenesis induction with dexamethasone, $\beta$ glycerophosphoric acid and vitamin $C$, and cardiomyocyte induction with 5-azacytidine. After 7 , 14, 21 and 28 days of induction, immunocytochemistry was performed to detect the expressions of type I and II collagen and osteocalcin, and alizarin red staining was performed to detect the bone nodule formation in osteogenesis induction. Immunocytochemistry was carried out to detect the striated muscle actin expression in cardiomyocytes. The hMSCs undergoing successful transfection were positive for the hTERT. The hTERT transfected cells were grown in vitro successfully and passaged for 136 generations. Results showed that these cells could be induced to differentiate into chondrocytes, bone and myocardial cells. Introduction of exogenous hTERT into hMSCs could achieve immortalized hMSCs with the potential of multi-directional differentiation. Thus, these cells could be applied as seed cells in tissue engineering.
\end{abstract}

Key words: transfection, cell immortalization, telomerase, bone marrow stromal cells

\section{INTRODUCTION}

Human bone marrow stromal cells (hMSCs) are easy to collect, rapidly proliferate and have potential of multi-directional differentiation. In recent years, increasing attention has been paid on the application of hMSCs in tissue engineering and genetic engineering (Richardson et al. 2010; Zellner et al. 2010). However, the hMSCs can be passaged for only 34 generations due to the senescence (Xin et al. 2010). Thus, these cells cannot meet the requirement for seed cells in biological engineering (Petersen and Niklason 2007, Richardson et al. 2010) and this significantly limits the wide application of hMSCs in basic and clinical studies. In the present study, human telomerase reverse transcriptase (hTERT) was transfected into hMSCs, which could activate the activity of telomerase and delay the aging process of hMSCs. Thus, hTERT transfected hMSCs become immortalized and remain the potential of multi-directional differentiation. These cells provide basis for subsequent basic and clinical studies on biological engineering.

*Author for correspondence: tangyunn@yeah.net 


\section{MATERIALS AND METHODS}

\section{Main Reagents}

The hMSCs were collected from a 6-year-old patient with developmental dysplasia of the hip for monoclonal culture. The pCIneo-hTERT was kindly provided by Dr Weinberg (USA) and contained full length hTERT cDNA, restriction sites (EcoR I and Sal I) and G418 resistance. LDMEM low glucose medium, trypsin and G418 (Gibco, Michigan, USA), fetal bovine serum (FBS, Hyclone, South Logan, USA), dexamethasone, transforming growth factor $\beta$ (TGF- $\beta$ ), vitamin $\mathrm{C}$ and 5-azacytidine (Sigma, USA), rabbit anti-human hTERT monoclonal antibody (Santa Cruz, California, USA), mouse anti-human osteocalcin monoclonal antibody and anti-human actin monoclonal antibody (BD, California, USA), rabbit anti-human type I/II collagen polyclonal antibody and kits for immunocytochemistry (Zhongshan Biotech Co., Ltd), primers for RT-PCR (Shanghai Jikang Biotech,), Access RT-PCR kit (Promega, San Luis Obispo, California, USA), LipofectamineTM 2000 kit for transfection (Invitrogen, USA), potable flask (50 mL, South Elgin, USA) and gel image analysis system (Shanghai Tianlong Instrument) were applied in the present study. Human embryonic kidney cells (HEK-293 cells) were kindly provided by the Department of Pathology of Forth Military Medical University.

\section{Complete medium}

L-DMEM contained 10\% FBS and $100 \mathrm{U} / \mathrm{mL}$ penicillin-streptomycin. The medium for the chondrogenesis induction was the complete medium containing dexamethasone $\left(1 \times 10^{-8}\right.$ $\mathrm{mol} / \mathrm{L})$, TGF- $\beta \quad(5 \mu \mathrm{g} / \mathrm{L})$ and vitamin $\mathrm{C}(10$ $\mathrm{mM} / \mathrm{L}$ ), medium for the osteogenesis induction was the complete medium containing dexamethasone $\left(1 \times 10^{-8} \quad \mathrm{mM} / \mathrm{L}\right), \quad$ glycerol phosphate $(50 \mathrm{mg} / \mathrm{L})$, vitamin $\mathrm{C}(10 \mathrm{mM} / \mathrm{L})$, and the medium for cardiomyocyte induction was the complete medium containing 5-azacytidine (3 $\mu \mathrm{M} / \mathrm{L})$.

\section{Cells for transfection}

The hMSCs were collected from the healthy subjects for monoclonal culture and two monoclonal colonies were obtained. The examination confirmed the potential of these cells to differentiate into bone, chondrocytes and myocardial cells. These cells had expressions of
CD29, CD59 and CD116 but had no expressions of CD34, CD45, CD117 or HLA-DR. One of the colonies was collected for subsequent transfection (Teng and Wang 2007).

\section{Transfection of pCIneo-hTERT into hMSCs}

The hMSCs were seeded into a 24-well plate $\left(1 \times 10^{5}\right.$ cells/well). When cell confluence reached $95 \%$, cells were transfected with pCIneo-hTERT with LipofectamineTM 2000 kit according to manufacturer's instructions. Passaging was done $24 \mathrm{~h}$ later at a ratio of 1:10. On the next day, cells were maintained in complete medium containing $0.2 \mathrm{~g} / \mathrm{L}$ G418. Five to seven days later, cells without resistance to G418 died. Then, the concentration of G418 in the medium was reduced to $0.1 \mathrm{~g} / \mathrm{L}$. Two to three weeks later, G418 resistant colony was collected and transferred into 96-well plate and 24-well plate for further culture. A total of 15 colonies had favorable growth. Then, six colonies with good growth were collected for culture and the remaining colonies were frozen for storage. In the subsequent culture, hMSCs of colony 3 had the best growth and then used for the construction of immortalized hMSCs. These cells were named as mesenchymal stem cell line-XiJin.

\section{Detection of hTERT in transfected cells}

1- Extraction of total RNA: Untransfected hMSCs of passage 7, 293 cells, and hMSCs of colony 3 of passage 13 and passage 100 were collected independently and total RNA was extracted by using Trizol.

2- RT-PCR: Mixture $(50 \mu \mathrm{L})$ for RT-PCR contained $2.0 \mu \mathrm{L}$ of template RNA, $0.2 \mathrm{mmol} / \mathrm{L}$ $\mathrm{dNTP}, 0.4 \mu \mathrm{mol} / \mathrm{L}$ primers, $5.0 \mathrm{Mmol} / \mathrm{L}$ DTT, 10 $\mu \mathrm{L}$ of RNasin, $1.5 \mathrm{Mmol} / \mathrm{L} \mathrm{MgCl} 2,1.0 \mu \mathrm{L}$ of enzyme Mix and $\mathrm{ddH}_{2} \mathrm{O}$. Conditions for the RTPCR were as follows: $50^{\circ}$.C for 30 min, 10 cycles of $94^{\circ} . \mathrm{C}$ for $10 \mathrm{~s}, 56^{\circ}$.C for $30 \mathrm{~s}$ and $68^{\circ} . \mathrm{C}$ for 45 $\mathrm{s}, 25$ cycles of $94^{\circ} \mathrm{C}$ for $10 \mathrm{~s}, 56^{\circ} \mathrm{C}$ for $30 \mathrm{~s}$ and $68^{\circ} \mathrm{C}$ for $2 \mathrm{~min}, 68^{\circ} \mathrm{C}$ for $7 \mathrm{~min}$.

3- Western blot assay: Cells were lysed with lysis buffer (Tris $\mathrm{HCl} 0.05 \mathrm{~mol} / \mathrm{L}, \mathrm{NaCl} 0.15 \mathrm{~mol} / \mathrm{L}$, $\mathrm{NaN}_{3} 0.2 \mathrm{~g} / \mathrm{L}$, SDS $1 \mathrm{~g} / \mathrm{L}, \mathrm{NP}-4010 \mathrm{~mL} / \mathrm{L}$ and PMSF $0.1 \mathrm{~g} / \mathrm{L}$ ) followed by extraction of the total protein and determination of protein concentration. Western blot assay was done to detect the protein expression of hTERT according to previous report (Sambrook 2002). A total of $50 \mu \mathrm{g}$ of proteins were subjected for the assay. Luminol chemiluminescence reaction was employed for visualization. 
4- Detection of telomerase activity: Telomerase activity was detected in the hMSCs of passage 13 before and after the transfection and in transfected hMSCs of passage 100. A total of 293 cells served as positive control and 293 cells followed the treatment at $65^{\circ} \mathrm{C}$ for $30 \mathrm{~min}$ as negative control. Telomerase activity was measured according to the manufacturer's instructions. The products following the amplification then underwent hybridization and visualization and absorbance (A) was measured at $450 \mathrm{~nm}$ reflecting the telomerase activity. Determination of the positive staining A of $<0.1$ was expressed as 0.1 and that of $>0.1$ as the actual value. Positivity was defined when the A of sample was 5-folds higher than that of negative control.

5- Immunocytochemistry for hTERT: Immunocytochemistry was performed to detect the hTERT expression in hMSCs of passage 13 by using SABC method.

\section{Osteogenesis induction}

The hTERT transfected hMSCs of colony 3 of the passage 100 were grown in the medium for osteogenesis induction for 7, 14, 21 and 28 days. The cells were collected and seeded into an acid pre-treated 24-well plate at a density of $2 \times 10^{5} / \mathrm{mL}$, followed by incubation for $36 \mathrm{~h}$. When cell confluence reached nearly $100 \%$, cells were collected and processed as follows: 1) cells were fixed in $40 \mathrm{~g} / \mathrm{L}$ paraformaldehyde for $20 \mathrm{~min}$. Type I collagen and osteocalcin were measured by immunocytochemistry using SABC method. Visualization was done with $\mathrm{DAB}$ for type I collagen and with immunofluorescene for osteocalcin; 2) Cells were fixed in $950 \mathrm{~mL} / \mathrm{L}$ alcohol for $15 \mathrm{~min}$ followed by alkaline phosphatase (ALP) assay with calcium-cobalt staining method; 3) For bone nodule detection, cells were seeded at a density of $2 \times 10^{6} / \mathrm{mL}$ followed by incubation for $24 \mathrm{~h}$. When cells adhered to the plate, they were maintained in conditioning medium for one week when bone nodules formed. Then, they were fixed in $40 \mathrm{~g} / \mathrm{L}$ paraformaldehyde for $20 \mathrm{~min}$, followed by alizarin red staining. Human osteoblasts served as positive controls.

\section{Chondrogenesis induction}

The hTERT transfected hMSCs of colony 3 of the passage 100 were grown in the medium for chondrogenesis induction for 7, 14, 21 and 28 days. The cells were collected and seeded into acid pre-treated coverslips in a 24 -well plate at a density of $2 \times 10^{5} / \mathrm{mL}$, followed by incubation for $36 \mathrm{~h}$. When cell confluence reached nearly $100 \%$, coverslips were obtained and cells were fixed in $40 \mathrm{~g} / \mathrm{L}$ paraformaldehyde for $20 \mathrm{~min}$, followed by $\mathrm{H} \& \mathrm{E}$ staining, toluidine blue staining, and immunocytochemistry for type II collagen (SABC method and visualization with DAB). Human chondrocytes of the knee served as positive controls.

\section{Cardiomyocyte induction}

The hTERT transfected hMSCs of colony 3 of the passage 100 were grown in the medium for cardiomyocyte induction for 7, 14, 21 and 28 days. The cells were collected and seeded into acid pretreated coverslips in 24-well plate at a density of $2 \times 105 / \mathrm{mL}$, followed by incubation for $36 \mathrm{~h}$. When cell confluence reached nearly $100 \%$, coverslips were obtained and cells were fixed in $40 \mathrm{~g} / \mathrm{L}$ paraformaldehyde for $20 \mathrm{~min}$, followed by eosin staining and immunocytochemistry for actin (alkaline phosphatase method). Human myocardial cells served as positive controls.

\section{RT-PCR assay}

Cells were collected before and at 7, 14, 21 and 28 $\mathrm{d}$ after induction and lysed in Trizol reagent for the extraction of total RNA. Reverse transcription of RNA into cDNA was performed according to manufacturer's instructions. For the cells undergoing osteogenesis induction, the mRNA expressions of human type I collagen (hCOL I) and osteocalcin (hOC) was measure and for the cells undergoing chondrogenesis induction, the mRNA expression of type II collagen (hCOL II) was measured. For the cells undergoing cardiomyocyte induction, the mRNA expression of ventricle specific myosin light chain MLC-2V was determined. The primers were as follows: hTERT: 5'-3': GACACACATTCCACAGGTCG, 3'-5': GACTCGACACCGTGTCACCTAC, hCOL I: 5'3':ATCCGCAGTGGCCTCCTAAT, 3'5':GCATCTCATAGTGCATCTGG, hCOL II: 5'3:CTGGCTCCCAACACTGCCA ACGTC, 3'-5': TCCTTTGGGTTTGCAACGGATTGT, hOC: 5'-3': CGCAGCCACCG AGACACCAT, 3'5':GGGCAAGGGCAAGGGGAAGA, MLC-2v: 5'-3':GCCAAGAAGC GGATAGAAGG, 3'5':CTGTGGTTCAGGGCTCAGTC. Following the RT-PCR assay, products were subjected to $1.5 \%$ agarose gel electrophoresis and bands were analyzed with a gel image analysis system. 


\section{RESULTS}

\section{Culture of hMSCs}

For the hMSCs after hTERT transfection, there was no evident cell death. After screening with G418 for $48 \mathrm{~h}$, cells began to die and the number of dead cells was significantly increased $72 \mathrm{~h}$ after G418 screening. Almost all the hMSCs without hTERT transfection died 7 days after G418 screening. The number of hTERT transfected hMSCs was also small and these cells were mainly located along the plate wall. About $\sim 10$ days after G418 screening, colonies formed. Then, the G418 concentration was reduced to $0.1 \mathrm{~g} / \mathrm{L}$ for further screening. Four days later, G418 resistant cell colonies were identified and subsequently transferred into a 24 well plate, a 6 well plate and a $35 \mathrm{~mm}$ flask for further culture. A total of 15 colonies had favorable growth and six of them were collected for culture and the remaining colonies were frozen for storage. Among six colonies, colonies 1,2 and 5 were passaged for 90 days (45 53 generations) and senescence and death were found. However, colonies 3, 4 and 6 still had favorable growth. Subsequently, colony 3 was collected for further culture. Cells in colony 3 were spindle, had contact inhibition and their proliferation was rapider than the untransfected hMSCs. The doubling time of these cells was 1.68 days and freezing-thawing had no influence on the cell growth. These cells were passaged for about 220 days (136 generations, one generation representing the increase in the number of cells by 1-fold).

\section{hTERT expression in hTERT transfected hMSCs}

The hTERT mRNA expression was found in 293 cells of colony 3 of the passage 13 and 100 but absent in hMSCs without hTERT transfection. It is suggested that cells of colony 3 had hTERT expression. Western blot assay showed a band at $125 \mathrm{kDa}$ in the cells of colony 3 , which was confirmed as hTERT protein. Detection of telomerase activity revealed that hTERT transfected hMSCs were positive for telomerase and these cells of passage 100 still had high telomerase activity (Fig. 1). Immunohistochemistry indicated that hTERT was expressed in the nucleus (Fig. 2).

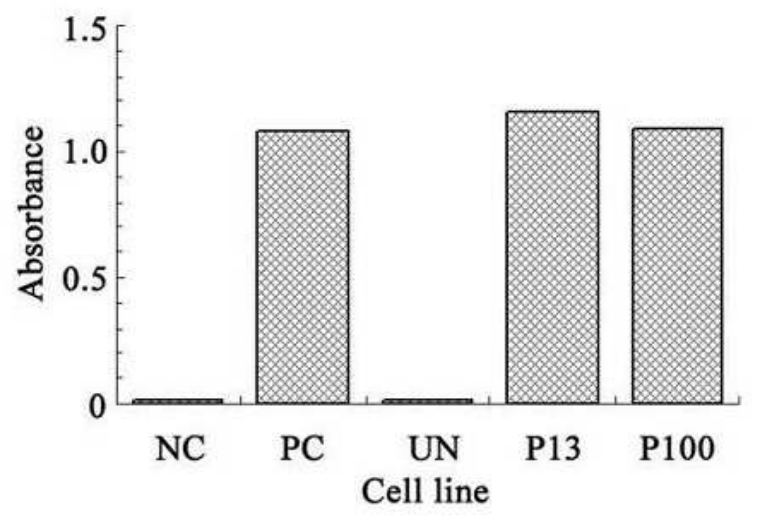

Figure 1 - Expression of activity of telomerase in transfected cells. NC, negative control (inactivate 293 cells), PC, positive control, (293 cells), UN, hMSCs, P13, MSCxj pf passage 13, P100, MSCxj of passage 100.

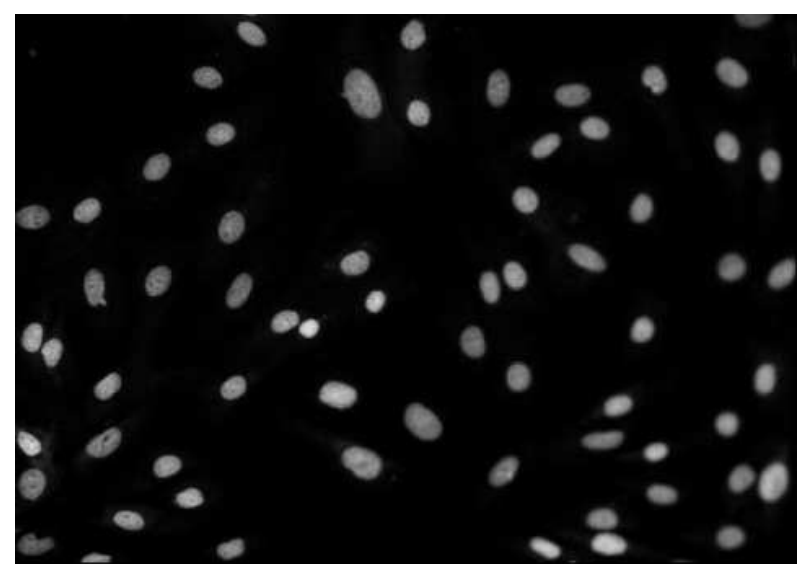

Figure 2 - Expression of hTERT with SABC staining $(\times 40)$.

\section{Osteogenesis induction}

Following osteogenesis induction, cells had no contact inhibition and further proliferated to form multiple cell layers and cell mass. In addition, crystal-like substance was also found. At 7, 14, 21 and $28 \mathrm{~d}$ after the induction, immunocytochemistry showed that the cells were positive for type I collagen and osteocalcin. Cells were positive for ALP and the positive rate increased over time and reached $95 \%$ at 24 days after the induction. The RT-PCR assay also revealed mRNA expressions of type I collagen and osteocalcin, which increased gradually (Fig 3). At 14, 21 and 28 days after the induction, alizarin red staining revealed bone nodules in the region with cell aggregation (Fig. 4). 


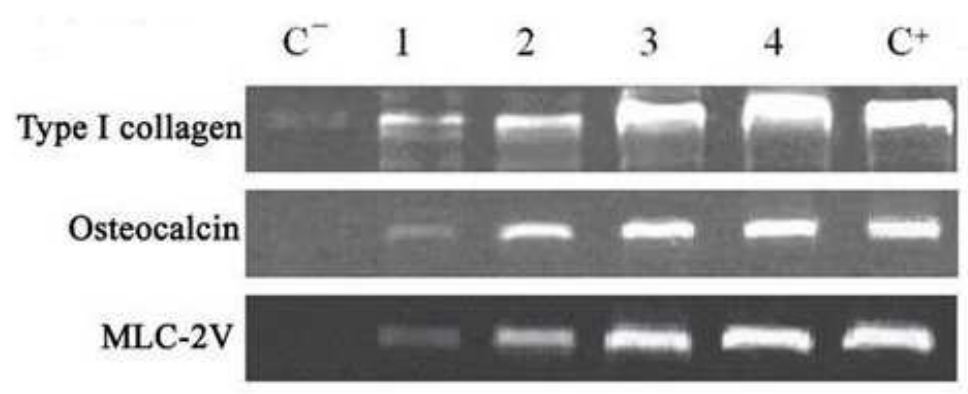

Figure 3 - The cells were induced into osteoblasts, and cadiocyte with the conditional culture mediums, and expressed mRNA of collogen I, osteocalcin and MLC-2V. N, negative control, 1/2/3/4, the induced cells at 1/2/3/4w, P, positive control.
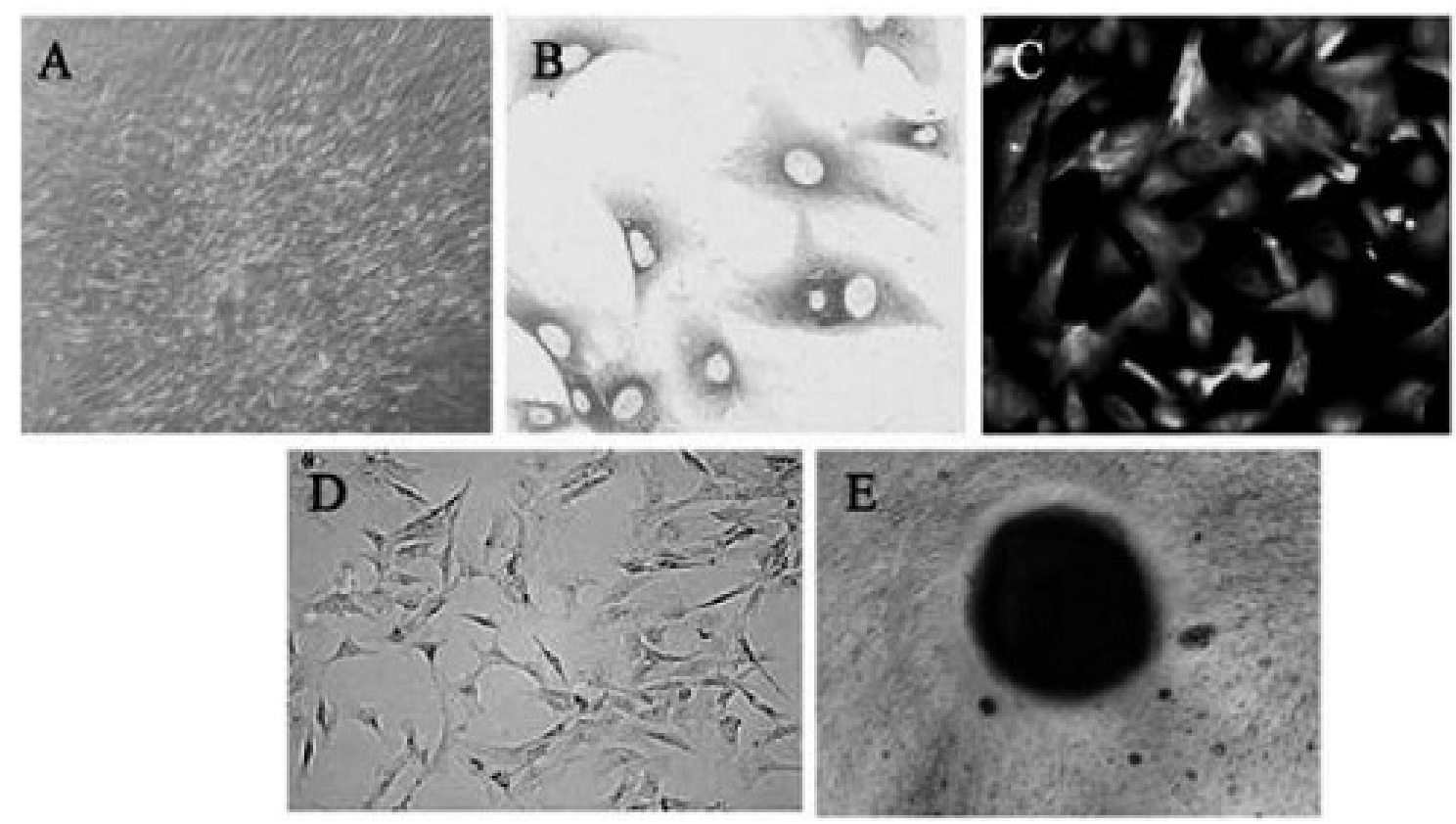

Figure 4 - The detection of osteoblast express of MSCxj of passage 100. A, the cells grew multilayer, agglomerate, crystallized and without contact inhibition, B, the express of collogen I, C, the expression of osteocalcin, D, positive reaction of alkaline, E, the formation of calcium nodus.

\section{Chondrogenesis induction}

At 7, 14, 21 and $28 \mathrm{~d}$ after the induction, the number of polyhedral cells increased. The RTPCR assay revealed the mRNA expression of type II collagen (Fig. 5). Immunocytochemistry showed the expression of type II collagen. In addition, cells were positive in toluidine blue staining and the positive rate increased over the time (Fig. 6).

\section{Cardiomyocyte induction}

One week after the induction, cells enlarged but were still long-spindle. Two weeks after the induction, cells became short-cylindrical and processes were present in both ends. Three weeks after the induction, cell processes contacted eachother and then were connected. Four weeks after the induction, cells connected and formed syncytium-like structure. Under a phase contrast microscope, spontaneous beating was noted (Fig. 7). Fourteen, 21 and 28 days after the induction, immunocytochemistry revealed actin expression and RT-PCR assay demonstrated mRNA expression of MLC-2V, which increased gradually (Fig. 3). 


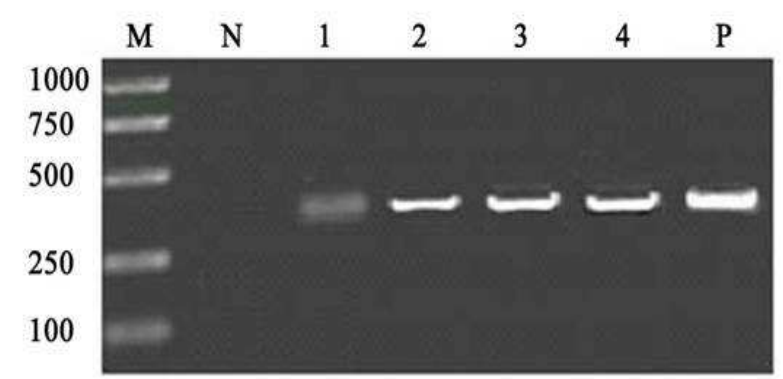

Figure 5 - The cells were induced into chondrocyte with the conditional culture mediums, and expressed mRNA of collogen I. M, Marker. N, negative control (hMSCs), 1/2/3/4, the induced cells at 1/2/3/4w, P, positive control (human chondrocyte of knee).

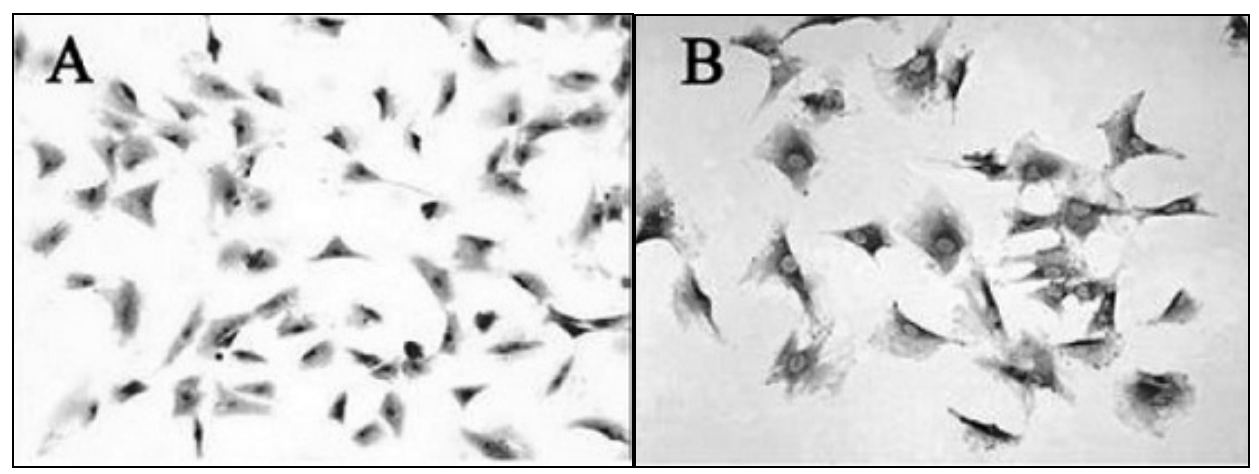

Figure 6 - The detection of chondrocyte express of MSCxj of passage 100. A, there was polygon and positive expression of toluidine blue, $\mathrm{B}$, expression of collogen I (SABC $\times 400)$.
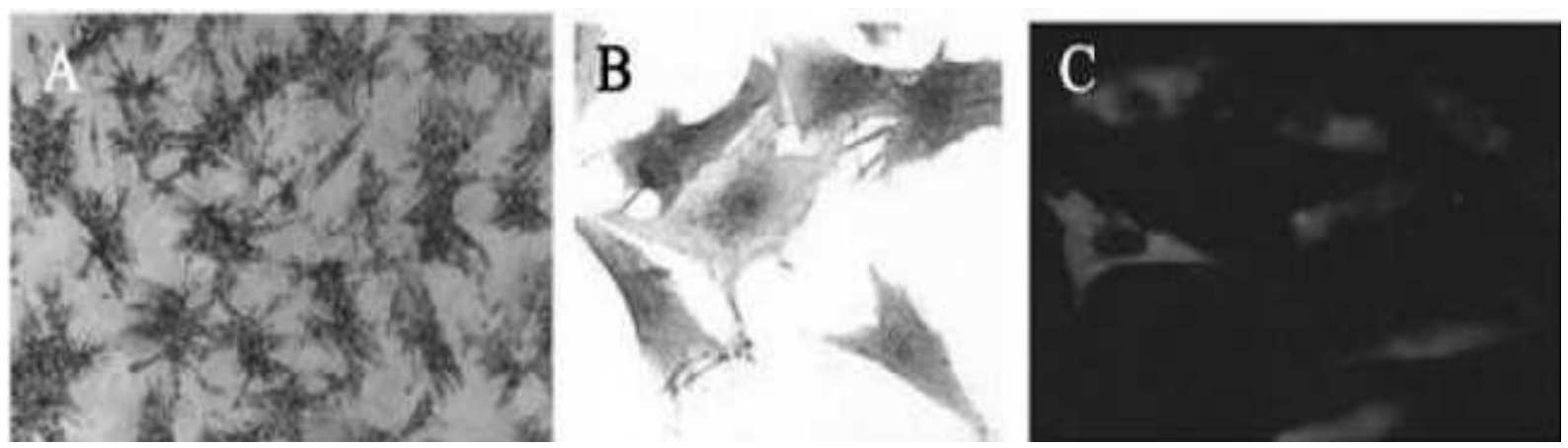

Figure 7 - The detection of cadiocyte expression of MSCxj of passage 100. A, the cells had symplasm construction, B, the cells' shape were stub structure, there were interconnect among the cells, and prominence at the end of the cells, C, Expression of actin.

\section{DISCUSSION}

MSCs belong to mononuclear cells locating in bone marrow and are adult stem cells with the potential of multiple-directional differentiation. MSCs are also known as marrow stromal stem cells and marrow mesenchymal stem cells (Richardson et al. 2010; Zellner et al. 2010). MSCs are rich in bone marrow and their collection is easy and safe. Thus, these cells have been usually collected for culture and analysis of gene modification. In addition, these cells have active 
proliferation and the potential of multipledirectional differentiation. Thus, increasing attention has been paid to the application of MSCs in biological engineering. However, in the tissue engineering, the number of MSCs cannot meet the requirement. In the basic researches, primary culture of MSCs is the frequently applied method and there is not standardized cell line for investigation. Therefore, the construction of immortalized human MSCs has important theoretical and practical values.

Cell immortalization refers to the characteristic of continuous proliferation of the cells (Satija et al. 2007). In several studies, exogenous virus, or oncogene has been introduced to target the cells to construct the immortalized cells (Kelekar and Cole 1987; Arimura et al. 2007; Wu et al. 2007), in which the integration of target gene was random and expression of target gene might have interfered with the intracellular physiological processes, which could result in unexpected changes such as loss of differentiation characteristic and lack of control of check point. The cells treated with virus, or oncogene belong to transformed cells but not the normal cells, and thus they are different partially, or completely from the normal cells in the transformation features such as changes in cell morphology, karyotype and tumorigenicity, as well as loss of suspended growth and contact inhibition (Gipson et al. 2007). The hTERT gene is an immortalization gene. If the exogenous hTERT could be transfected into the target cells and expressed stably, the reverse transcription activity of telomerase would increase to promote the reverse transcription of telomerase RNA. In addition, hTERT can also protect, or stabilize the telomerase RNA to prolong the half life of telomerase RNA (Petersen and Niklason 2007). The hTERT gene is an immortalization gene and exogenous hTERT can activate the activity of telomerase in target cells and maintain the length of telomere. Then, cells can pass the senescence phase (M1 phase) and crisis phase (M2 phase), leading to immortalization (Petersen and Niklason. 2007). Bodnar et al (1998) found that transient expression of the hTERT in normal diploid cells could activate the telomerase activity and prolong the cell survival. The hTERT has been successfully introduced to human periodontal ligament cells, osteoblasts, mammary epithelial cells and Spinal arachnoid cell line, (Xiaoxue et al. 2004; Zhao et al. 2010; Hasegawa et al. 2010; Janson et al. 2011). Different from the traditional immortalization gene, the process that TERT activates telomerase to induce the immortalization is similar to the physiological process in germline stem cells. Thus, hTERT transfected cells with immortalization preserve more biological characteristics of normal cells. Shay and Wright (2000) also confirmed that the transfection of exogenous hTERT not only led to cell immortalization, but had no influence on normal functions of the, which were critical in tissue engineering.

\section{CONCLUSIONS}

In the present study, exogenous hTERT gene was transfected into hMSCs, which then underwent screening with G418. A total of 15 cell colonies were collected. Three colonies had favorable growth in vitro and contact inhibition, their doubling time was 1.68 days and freezing-thawing had no influence on cell growth. These cells have been passaged for 136 generations (about 220 days). In addition, immortalized cells preserved the potential of multiple-directional differentiation of the primary cells and have been applied in several labs. These cells can serve as seed cells in tissue engineering. The cells transfected with the hTERT have the biological characteristics of normal cells and this characteristic is different from that of the cells undergoing transfection with exogenous virus, or oncogenes. Due to the difference in the gene transfection, cells might develop de-differentiation, malignant transformation, or loss of exogenous gene (Teng and Wang 2011), and further studies should be carried out.

\section{ACKNOWLEDGEMENTS}

This study was funded by the National Natural Science Foundation of China (No.31360229, No. 51165044) and "Twelve-five Year" Medical Research Project of Chinese PLA (No. CWS11J011). 


\section{REFERENCES}

Arimura K, Nakagawa M, Izumo S, Usuku K, Itoyama Y, Kira J, et al. Safety and efficacy of interferonalpha in 167 patients with human T-cell lymphotropic virus type 1-associated myelopathy. J Neurovirol. 2007; 13: 364-372.

Bodnar AG, Ouellette M, Frolkis M, Holt SE, Chiu CP, Morin GB, et al. Extension of life-span by introduction of telomerase into normal human cells. Science. 1998; 279: 349-352.

Gipson CL, Xin ZT, Danzy SC, Parslow TG, Ly H. Functional characterization of yeast telomerase RNA dimerization. J Biol Chem. 2007; 282:18857-18863.

Hasegawa T, Chosa N, Asakawa T, Yoshimura Y, Ishisaki A, Tanaka M. Establishment of immortalized human periodontal ligament cells derived from deciduous teeth. Int J Mol Med. 2010; 26:701-705.

Janson C, Romanova L, Hansen E, Hubel A, Lam C. Immortalization and functional characterization of rat arachnoid cell lines. Neuroscience. 2011; 177:23-34.

Kelekar A, Cole MD. Immortalization by c-myc, H-ras, and Ela oncogenes induces differential cellular gene expression and growth factor responses. Mol Cell Biol. 1987;7:3899-38907. 3899-3907

Petersen T, Niklason L. Cellular lifespan and regenerative medicine. Biomaterials. 2007; 28: 3751 3756.

Richardson SM, Hoyland JA, Mobasheri R, Csaki C, Shakibaei M, Mobasheri A. Mesenchymal stem cells in regenerative medicine: opportunities and challenges for articular cartilage and intervertebral disc tissue engineering. J Cell Physiol. 2010; 222: 2332.

Sambrook J RD, ed. Molecular Cloning: A Laboratory Manual. 3rd Edition ed: Beijing: Science Press, 2002.
Satija NK, Gurudutta GU, Sharma S, Afrin F, Gupta P, Verma YK, et al. Mesenchymal stem cells: molecular targets for tissue engineering. Stem Cells Dev. 2007; 16:7-23.

Shay JW, Wright WE. The use of telomerized cells for tissue engineering. Nat Biotechnol. 2000; 18: 22-23.

Teng Y, Wang Z. Biological characteristics of human trabecular bone-derived stromal cells. Chin J Exp Surg. 2007; 24: 1411-1413.

Teng Y, Wang Z. The transformational phenotype of the immortalized human bone marrow stromal stem cell line MSCxj. Chin J Bone Tumor Bone Dis. 2011; 10: 586-589.

Wu Y, Maruo S, Yajima M, Kanda T, Takada K. Epstein-Barr virus (EBV)-encoded RNA 2 (EBER2) but not EBER1 plays a critical role in EBV-induced B-cell growth transformation. J Virol. 2007; 81: 11236-11245.

Xiaoxue Y, Zhongqiang C, Zhaoqing G, Gengting D, Qingjun M, Shenwu W. Immortalization of human osteoblasts by transferring human telomerase reverse transcriptase gene. Biochem Biophys Res Commun. 2004; 315: 643-651.

Xin Y, Wang YM, Zhang H, Li J, Wang W, Wei YJ, et al. Aging adversely impacts biological properties of human bone marrow-derived mesenchymal stem cells: implications for tissue engineering heart valve construction. Artif Organs. 2010; 34:215-222.

Zellner J, Mueller M, Berner A, Dienstknecht T, Kujat R, Nerlich M, et al. Role of mesenchymal stem cells in tissue engineering of meniscus. J Biomed Mater Res A. 2010; 94:1150-1161.

Zhao CF, Hu HY, Meng L, Li QQ, Lin AX. Immortalization of bovine mammary epithelial cells alone by human telomerase reverse transcriptase. Cell Biol Int. 2010; 34: 579-586. 